\title{
Markov Quantum Semigroups Admit Covariant Markov $C^{*}$-Dilations
}

\section{Jean-Luc Sauvageot}

Laboratoire de Probabilites, Universite Pierre et Marie Curie, 4 Place Jussieu, F-75230 Paris Cedex 05, France

\begin{abstract}
Through a Daniell-Kolmogorov type construction, to any Markov quantum semigroup on a $C^{*}$-algebra there is associated a quantum stochastic process which is a dilation of the semigroup, and satisfies a covariance rule which implies the weak Markov property.
\end{abstract}

\section{Introduction}

In the classical framework, a Markov semigroup is a semigroup $\left(P_{t}\right)_{t \geqq 0}$ of probability transitions on $a$ ( $n$ eventually compact) space $X$. The DaniellKolmogorov construction (cf. [3, Sect. I.2]) is a natural procedure for associating to such a semigroup a strong Markov process which dilates it.

The simplest way of viewing this construction is to build a family $\left(\mu^{x}\right)_{x \in X}$ of probability measures on the space $X^{\mathbb{R}+}$ of all (borel) trajectories as an inductive limit of the measures $\mu_{t_{1}, \ldots, t_{n}}^{x}$ on $X^{\left\{t_{1}, \ldots, t_{n}\right\}}\left(\mathrm{t}_{1}<\ldots<t_{n}\right)$ defined by

$$
\mu_{t_{1}, \ldots, t_{n}}^{x}(f)=\int_{X} f\left(x_{1}, \ldots, x_{n}\right) P_{t_{n}-t_{n-1}}\left(x_{n-1}, d x_{n}\right) \ldots P_{t_{2}-t_{1}}\left(x_{1}, d x_{2}\right) P_{t_{1}}(x, d x) .
$$

More algebraically, consider the $P_{t}$ as positive maps from $C(X)$ (the algebra of continuous functions on $X$ ) into itself, assuming they preserve the class of continuous functions. For any $t$ choose a $C^{*}$-algebra $A_{t}$ isomorphic to $C(X)$; then, for $t_{1}<t_{2}$, consider the conditional expectation $\varepsilon_{t_{2}, t_{1}}$ from $A_{t_{1}} \otimes A_{t_{2}}$ onto $A_{t_{1}}$ characterized by

$$
\varepsilon_{t_{2}, t_{1}}\left(f_{2} \otimes f_{1}\right)=P_{t_{2}-t_{1}}\left(f_{2}\right) f_{1}
$$

On the tensor product $A_{t_{n}} \otimes \ldots \otimes A_{t_{1}}$, one defines a family $\left(E_{t_{k}}\right)_{k=1, \ldots, n}$ of conditional expectations onto the sub $C^{*}$-algebra $A_{t_{k}} \otimes \ldots \otimes A_{t_{1}}$ through the induction formula

$$
E_{t_{k}}=\left(\varepsilon_{t_{k+1}, t_{k}} \otimes \text { identity }\right) \circ E_{t_{k+1}} .
$$

The Markov process lies in the inductive limit of the $C^{*}$-algebras $A_{t_{n}} \otimes \ldots \otimes A_{t_{1}}$ along the filter of finite subsets $\left\{t_{1}, \ldots, t_{n}\right\}$ of $\mathbb{R}_{+}$. Its filtration is given by the inductive limit of the $E_{t}\left(t \in\left(t_{1}, \ldots, t_{n}\right\}\right)$, and a time evolution $\left(\sigma_{s}\right)_{s \geqq 0}$ is provided by 
the canonical isomorphisms of $A_{t_{n}} \otimes \ldots \otimes A_{t_{1}}$ with $A_{t_{n}+s} \otimes \ldots \otimes A_{t_{1}+s}$. The filtration and the time-evolution are linked by the covariance rule:

$$
\sigma_{s} \circ E_{t}=E_{t+s} \circ \sigma_{s}, \quad \forall s, t \geqq 0 .
$$

One should notice that this covariance property is a step towards the strong Markov property, which reads

$$
\sigma_{s} \circ E_{\tau}=E_{\tau+s} \circ \sigma_{s}
$$

for any stopping time $\tau$ [although (0.2) involves only the stopping times $\tau=t$ ]. Written for $t=0$, it implies the weak Markov property of conditional independence of the future with the past, given the present (cf. our comment after Theorem 3.1 of this paper). Together with additional assumptions for instance whenever almost all trajectories in the process are right continuous, the covariance formula (0.2) actually implies strong Markov property.

In the $C^{*}$-algebraic framework, a Markov quantum semigroup $\left(\phi_{t}\right)_{t \geqq 0}$ is defined on a $C^{*}$-algebra with unit $A$ : it is a semigroup of completely positive maps from $A$ into itself which satisfy $\phi_{t}\left(1_{A}\right)=1_{A}, \forall t \geqq 0$; no continuity requirement is assumed.

It has been for a long time an open question whether such a quantum semigroup could always be dilated by a Markov quantum process. After Evans and Lewis showed that it could be dilated by a semigroup of $*$-algebraic endomorphisms of a larger algebra [4], Accardi [1] attempted to repeat the Daniell-Kolmogorov construction with a loss of the conditional expectation property in (0.1) above. Only recently the existence of Markov dilations was proved by Hudson and Parthasarathy [5] with analytical assumptions on the type of the infinitesimal generator of the semigroup. (For further bibliography, for the terminology and the physical relevance of this problem, cf. Accardi [2], and the Lecture Notes n. 1055 in which [5] is published.) The problem is also stated and nearly solved, but only for von Neumann algebras, in [8]. (I am indebted to the referee for having pointed out to me this reference.)

In this paper, the problem is solved in full generality: to any Markov semi-group is associated, through a Daniell-Kolmorov type construction, a quantum dilation which satisfies the covariance property $(0.2)$ above, and thus is a Markov process. The precise statement is detailed as Theorem 3.1.

The main difficulty we had to face, referring to formula (0.1) above, is the nonpositivity of a product $\phi_{t}\left(a_{2}\right) a_{1}$ when both $a_{1}$ and $a_{2}$ are positive elements of $A$. In other words, as soon as the $C^{*}$-algebra $B$ is non-abelian, there is no natural way to write a completely positive map from $A$ into $B$ as the composition of a representation of $A$ in a larger $C^{*}$-algebra containing $B$, with a conditional expectation of this algebra onto $B$. The first section of the paper is devoted to a solution of this problem: to a pair $(A, B)$ of $C^{*}$-algebras with unit and a completely positive map $\phi$ from $A$ into $B$, with $\phi\left(1_{A}\right)=1_{B}$, is associated a $C^{*}$-algebra $A *_{\phi} B$ which is generated by two representations $\left\{a \rightarrow a *_{\phi} 1_{B}\right\}$ and $\left\{b \rightarrow 1_{A} *_{\phi} b\right\}$ of $A$ and $B$ respectively, and a conditional expectation $E_{\phi}$ from $A{ }_{\phi} B$ onto the range of $B$ satisfying

$$
E_{\phi}\left(a *_{\phi} 1_{B}\right)=1_{A} *_{\phi} \phi(a), \quad \forall a \in A
$$


This construction, which is a mixture of Stinespring's construction [6] with the notion of free product of $C^{*}$-algebras (cf. [7]), will be canonical up to the choice of an auxiliary state on $B$; and Sect. 2 is devoted to functorial properties of this "amalgamated free product" $A *_{\phi} B$, in order to be able to iterate it.

In Sect. 3, the problem is solved as Theorem 3.1: those properties allow us to associate to a finite subset $\Gamma=\left\{t_{1}, \ldots, t_{n}\right\}$ of $\mathbb{R}_{+}$a $C^{*}$-algebra

$$
\mathfrak{U}_{\Gamma}=\left(\ldots\left(\left(A *_{\phi_{t_{n}-t_{n-1}}} A\right) *_{\phi_{t_{n-1}-t_{n-2}}} A\right) * \ldots\right) *_{\phi_{t_{2}-t_{1}}} A,
$$

together with conditional expectations $\left(E_{t}, t \in \Gamma\right)$ of $\mathfrak{A}_{\Gamma}$ onto its sub $C^{*}$-algebra $\mathfrak{A}_{\Gamma \cap[0, t]}$. The $\left(\mathfrak{A}_{\Gamma}, E_{t}\right)$ form an inductive system and, as explained for the classical case, the inductive limit provides the covariant quantum Markov process which dilates

$$
\left(\phi_{t}\right)_{t \geqq 0} .
$$

All this construction can be repeated in the $W^{*}$-algebraic framework, just by considering $\sigma$-weak closures wherever we consider norm closures, and Theorem 3.1 can be stated for von Neumann algebras, all the morphisms (states, completely positive maps, conditional expectations, *-endomorphisms) being then normal. Our paper can then be considered as an improvement of the results of [8].

Some further comments on the result: our construction is a rather rough one, and highly non-commutative. In order to develop a satisfactory theory of stopping times (which are the main tool for studying stochastic processes, together with the supermartingale theorem which is still missing in the non-commutative case), we need at least two more properties:

- when imbedding the $C^{*}$-algebra $A$ in the bigger $C^{*}$-algebra $\mathfrak{A}$, where the quantum process lies, one should expect that the center of $A^{\prime \prime}$, or at least part of it (the ideal center) should be imbedded in the center of $\mathfrak{A}^{\prime \prime}$;

- continuity properties of the quantum semi-group $\left(\phi_{t}\right)_{t \geqq 0}$ should imply continuity properties in the dilation, for instance right continuity of the filtration; none of them is satisfied in our construction.

We know how to remedy those two deficiencies separately, by adapting what we have done here. However, a satisfactory theory of Markov quantum dilations will be developed only when they are solved together.

\section{An Amalgamated Quasi-Free Product of $C^{*}$-Algebras}

1.1. We are dealing with the following objects:

- two $C^{*}$-algebras with unit, $A$ and $B$

- a completely positive map $\phi: A \rightarrow B$ which respects the units: $\phi\left(1_{A}\right)=1_{B}$

- an auxiliary state $\omega$ on $B$ (on which no special requirement will be made).

1.2. We start with a concrete realization of $(\phi, \omega)$, that is a triple $(\Omega, H, K)$ where

- $K$ is a Hilbert space together with a (non-degenerate) representation of $A$ in $L(K): K$ will be written as a left $A$-module.

- $H$ is a closed subspace of $K$, imbedded with a representation of $B$ ( $H$ will be written as a left $B$-module), such that

$$
\left\langle a \xi_{1}, \xi_{2}\right\rangle=\left\langle\phi(a) \xi_{1}, \xi_{2}\right\rangle, \forall \xi_{1}, \xi_{2} \in H \subset K, \forall a \in A .
$$


- $\Omega$ is a unit vector in $H$ implementing $\omega$ :

$$
\langle b \Omega, \Omega\rangle=\omega(b), \quad \forall b \in B .
$$

For applications, we have:

$$
\langle a b \Omega, \Omega\rangle=\omega(\phi(a) b), \quad \forall a \in A, \quad \forall b \in B .
$$

(such a triple exists with arbitrary $H$ by Stinespring construction. Notice that to $K$ can be added any left $A$-module).

1.3. We shall adopt the following notations:

$$
\begin{aligned}
& H^{-}=H \ominus \mathbb{C} \Omega \quad \text { (orthogonal complement of } \Omega \text { in } H \text { ) } \\
& L=K \ominus H \quad \text { (orthogonal complement of } H \text { in } K \text { ) } \\
& L^{+}=K \ominus H=L \oplus \mathbb{C} \Omega \\
& L^{\prime}=K \ominus \overline{A H} \quad \text { (orthogonal complement in } K \text { of the cyclic span of } H \\
& \text { with respect to the action of } A \text { ) }
\end{aligned}
$$

$$
L^{+}=K \ominus \overline{A H}^{-} .
$$

We have $L^{\prime} C L, L^{\prime} C L^{+} C L^{+}$and, in most cases, $L^{\prime}=L^{++}$(but we won't need it). $L^{+\otimes \mathbb{N}}$ will be the infinite tensor product of countably many copies of $L^{+}$, with respect to the unit vector $\Omega \in L^{+}$.

We shall consider

- the vacuum vector $\tilde{\Omega}=\Omega^{\otimes \mathbb{N}}$,

- the creation operators $l(\eta)$ :

$$
l(\eta)\left[\eta_{1} \otimes \ldots \otimes \eta_{n} \otimes \widetilde{\Omega}\right]=\eta \otimes \eta_{1} \otimes \eta_{2} \otimes \ldots \otimes \eta_{n} \otimes \widetilde{\Omega}
$$

$\left(\eta \in L^{+} ; \eta_{1} \otimes \ldots \otimes \eta_{n} \otimes \widetilde{\Omega}\right.$ is the generic vector of $\left.L^{+\otimes \mathbb{N}}\right)$.

(1.3.1) the canonical isomorphism of $L^{+} \otimes L^{+\otimes \mathbb{N}}$ with $L^{+\otimes \mathbb{N}}$, which to the elementary tensor $\eta \otimes \widetilde{\zeta}\left[\eta \in L^{+}, \widetilde{\zeta} \in L^{+\otimes \mathbb{N}}\right]$ associates $l(\eta) \widetilde{\zeta}$.

(1.3.2) the decomposition $L^{+\otimes \mathbb{N}}=\mathbb{C} \tilde{\Omega} \oplus L \bigoplus_{n \geqq 1}\left(L^{+\otimes n} \otimes L\right)$ of $L^{+\otimes \mathbb{N}}$, obtained by

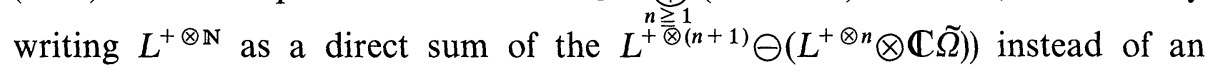
increasing limit.

\subsection{We set}

$$
K *^{\Omega} H=H \otimes L^{+\otimes \mathbb{N}}
$$

which is a left $-B$-module, as an ampliation of the left $B$-module $H$.

(1.4.1) We consider the canonical isomorphism $W: K *^{\Omega} H \rightarrow K \otimes L^{+\otimes \mathbb{N}}$ given by the following chain of identifications:

$$
\begin{aligned}
H \otimes L^{+\otimes \mathbb{N}} & =\left(H^{-} \otimes L^{+\otimes \mathbb{N}}\right) \oplus\left(\mathbb{C} \Omega \otimes L^{+\otimes \mathbb{N}}\right) \\
& \approx\left(H^{-} \otimes L^{+\otimes \mathbb{N}}\right) \oplus\left(L^{+\otimes \mathbb{N}}\right) \\
& \approx\left(H^{-} \otimes L^{+\otimes \mathbb{N}}\right) \oplus\left(L^{+} \otimes L^{+\otimes \mathbb{N}}\right) \\
& \approx\left(H^{-} \oplus L^{+}\right) \otimes L^{+\otimes \mathbb{N}} \\
& =K \otimes L^{+\otimes \mathbb{N}},
\end{aligned}
$$


so that $K *^{\Omega} H$ is imbedded with a structure of left $A$-module, as isomorphic to an ampliation of $K$.

Our purpose is to show that the $C^{*}$-algebra $A *_{\phi}^{\omega} B$ generated by the ranges of $A$ and $B$ respectively in $K *^{\Omega} H$ does not depend on the particular choice of $(\Omega, H, K)$, as soon as $H$ and $K$ are "big enough;" that there is a conditional expectation onto the range of $B$ whose restriction to $A$ is $\phi$; and that the construction has functional properties which allow us to iterate it.

1.5. Writing $H=H^{-} \oplus \mathbb{C} \Omega$, and decomposing $L^{+\otimes \mathbb{N}}$ as in 1.3.2, we get

$$
K *^{\Omega} H=H \oplus L \oplus\left(H^{-} \otimes L\right) \oplus \ldots \oplus\left(L^{+\otimes n} \otimes L\right) \oplus\left(H^{-} \otimes L^{+\otimes n} \otimes L\right) \oplus \ldots .
$$

From this decomposition, the structure of $A$-module on $K *^{\Omega} H$ can be recovered as follow: the sum $H \oplus L \approx K$ of the first two components is mapped by $W$ onto the subspace $K \otimes \mathbb{C} \widetilde{\Omega}$ of $K \otimes L^{+\otimes \mathbb{N}}$; gathering two by two the other components, we get $A$-invariant subspaces of $K *^{\Omega} H$

$$
\left(H^{-} \otimes L^{+\otimes n} \otimes L\right) \oplus\left(L^{+\otimes(n+1)} \otimes L\right) ;
$$

the restriction of $W$ is given by trivial identifications:

$$
\begin{aligned}
\left(H^{-} \otimes L^{+\otimes n} \otimes L\right) \oplus\left(L^{+\otimes(n+1)} \otimes L\right) & \approx\left(H^{-} \otimes L^{+\otimes n} \otimes L\right) \oplus\left(L^{+} \otimes L^{+\otimes n} \otimes L\right) \\
& \approx\left(H^{-} \oplus L^{+}\right) \otimes L^{+\otimes n} \otimes L \\
& \approx K \otimes L^{+\otimes n} \otimes L C K \otimes L^{+\otimes \mathbb{N}} .
\end{aligned}
$$

(1.5.1) $w_{H}$ will denote the natural isomorphism of $H$ with the first component of $K *^{\Omega} H$ written as above. We have

$$
\begin{aligned}
w_{H} \xi=\xi \otimes \tilde{\Omega} \in H \otimes L^{+\otimes \mathbb{N}}, & \forall \xi \in H \\
W w_{H} \xi & =\xi \otimes \tilde{\Omega} \in K \otimes L^{+\otimes \mathbb{N}}, \quad \forall \xi \in H .
\end{aligned}
$$

(1.5.2) $b \rightarrow 1_{A} *_{\phi}^{\omega} b$ will denote the natural representation of $B$ in

$$
\mathscr{L}\left(K *^{2} H\right)=\mathscr{L}(H) \otimes \mathscr{L}\left(L^{+\otimes \mathbb{N}}\right) .
$$

$a \rightarrow a *_{\phi}^{\omega} 1_{B}$ will denote the natural represenation of $A$ in

$$
\mathscr{L}\left(K *^{\Omega} H\right)=W^{*}\left[\mathscr{L}(K) \otimes \mathscr{L}\left(L^{+\otimes \mathbb{N}}\right)\right] W .
$$

We have

$$
w_{H}^{*}\left(a *_{\phi}^{\omega} 1_{B}\right) w_{H}=\phi(a), \quad \forall a \in A .
$$

1.6. Lemma. Let $\zeta$ in $K *^{\Omega} H$ (decomposed as in 1.5 above) be in a component $L^{+\otimes n} \otimes L, n \geqq 0$.

$\left.1^{\circ}\right)$ Let $a$ be in $A$, and $b$ in $B$ such that $\omega(b)=0$. Then

$$
\left(a *_{\phi}^{\omega} 1_{B}-1_{A} *_{\phi}^{\omega} \phi(a)\right)\left(1_{A} *_{\phi}^{\omega} b\right) \zeta
$$

belongs to the sum $\left(L^{+\otimes n} \otimes L\right) \oplus\left(L^{+\otimes(n+1)} \otimes L\right)$ and is equal to

$$
\left(p_{L^{+}} a b \Omega \otimes \zeta\right)-\omega(\phi(a) b) \zeta
$$

(where $p_{L^{+}}$denotes the orthogonal projection on $L^{+}$). 
$\left.2^{\circ}\right)$ Let $k \geqq 1, a_{1}, \ldots, a_{k} \in A, b_{1}, \ldots, b_{k} \in B$ such that $\omega\left(b_{1}\right)=\ldots=\omega\left(b_{k}\right)=0$. Then the vector

$$
\zeta_{k}=\left(a_{k} *_{\phi}^{\omega} 1_{B}-1_{A} *_{\phi}^{\omega} \phi\left(a_{k}\right)\right)\left(1_{A} *_{\phi}^{\omega} b_{k}\right) \ldots\left(a_{1} *_{\phi}^{\omega} 1_{B}-1_{A} *_{\phi}^{\omega} \phi\left(a_{1}\right)\right)\left(1_{A} *_{\phi}^{\omega} b_{1}\right) \cdot \zeta
$$

belong to $\bigoplus_{j=0}^{k}\left(L^{+\otimes(n+j)} \otimes L\right)$, and we have

$$
\left\langle\zeta_{k}, \zeta\right\rangle=(-1)^{k} \prod_{j=1}^{k} \omega\left(\phi\left(a_{j}\right) b_{j}\right)\langle\zeta, \zeta\rangle .
$$

Proof. $1^{\circ}$ ) For making $B$ to act on $\zeta$, we identify $\zeta \in L^{+\otimes n} \otimes L$ with $\Omega \otimes \zeta \in \mathbb{C} \Omega \otimes L^{+\otimes n} \otimes L$ as a vector in

$$
H \otimes L^{+\otimes n} \otimes L=\left(\mathbb{C} \Omega \otimes L^{+\otimes n} \otimes L\right) \oplus\left(H^{-} \otimes L^{+\otimes n} \otimes L\right),
$$

and, because of $\omega(b)=0,\left(1_{A} *_{\phi}^{\omega} b\right) \zeta$ is the vector $b \Omega \otimes \zeta$ in $H^{-} \otimes L^{+\otimes n} \otimes L$; $\left(1_{A} *_{\phi}^{\omega} \phi(a) b\right) \zeta$ is the vector $\omega(\phi(a) b) \zeta+p_{H^{-}} \phi(a) b \Omega \otimes \zeta$ in

$$
\left(L^{+\otimes n} \otimes L\right) \oplus\left(H^{-} \otimes L^{+\otimes n} \otimes L\right) .
$$

For making $A$ to act, we identify $H^{-} \otimes L^{+\otimes n} \otimes L$ with a part of $K \otimes L^{+\otimes n} \otimes L$, and we get

$$
\begin{aligned}
\left(a *_{\phi}^{\omega} 1_{B}\right)\left(1_{A} *_{\phi}^{\omega} b\right) \zeta & =\left(a *_{\phi}^{\omega} 1_{B}\right)(b \Omega \otimes \zeta) \\
& =\left(p_{H^{-}} a b \Omega \otimes \zeta\right) \oplus\left(p_{L^{+}} a b \Omega \otimes \zeta\right) \\
& =\left(p_{H^{-}} \phi(a) b \Omega \otimes \zeta\right) \oplus\left(p_{L^{+}} a b \Omega \otimes \zeta\right)
\end{aligned}
$$

$2^{\circ}$ ) is obtained by iterating $1^{\circ}$ ).

1.7. Proposition and Definition. Let $(\Omega, H, K)$ be as in 1.2 , with the following properties:

(i) The B-module $H$ is faithful (i.e. $b \in B, b \xi=0, \forall \xi \in H \Rightarrow b=0$ ).

(ii) The A-module $L^{\prime}=K \ominus \overline{A H}$ is faithful.

Let $A *_{\phi}^{\omega} B$ be the $C^{*}$-algebra in $L\left(K *^{\Omega} H\right)$ generated by the (faithful) representations $\left\{A \ni a \rightarrow a *_{\phi}^{\omega} 1_{B}\right\}$ and $\left\{B \ni b \rightarrow 1_{A} *_{\phi}^{\omega} b\right\}$. Then:

(1.7.1) There exists a (unique) conditional expectation $E_{\phi}^{\omega}$ from $A *_{\phi}^{\omega} B$ onto the range $\mathbb{C}_{A} *_{\phi}^{\omega} B$ of $B$, such that

- $E_{\phi}^{\omega}\left[a *_{\phi}^{\omega} 1_{B}\right]=1_{A} *_{\phi}^{\omega} \phi(a), \quad \forall a \in A$

. $\forall n \geqq 1, \quad \forall a_{1}, \ldots, a_{n} \in A, \quad \forall b_{1}, \ldots, b_{n} \in B$

with $\omega\left(b_{2}\right)=\ldots=\omega\left(b_{n}\right)=0$, then

$$
E_{\phi}^{\omega}\left[\left(a_{n} *_{\phi}^{\omega} 1_{B}-1_{A} *_{\phi}^{\omega} \phi\left(a_{n}\right)\right)\left(1_{A} *_{\phi}^{\omega} b_{n}\right) \ldots\left(a_{1} *_{\phi}^{\omega} 1_{B}-1_{A} *_{\phi}^{\omega}\left(a_{1}\right)\right)\left(1_{A} *_{\phi}^{\omega} b_{1}\right)\right]=0 .
$$

(1.7.2). The $C^{*}$-algebra $A *_{\phi}^{\omega} B$ (with the representations which generate it, and the conditional expectation $\left.E_{\phi}^{\omega}\right)$ does not depend on the particular choice of $(\Omega, H, K)$ satisfying specifications (i) and (ii) above.

Proof of the Proposition.

1) Write, for $k=1, \ldots, n$ and $\xi$ in $H$

- $Y_{k}=\left(a_{k} *_{\phi}^{\omega} 1_{B}-1_{A} *_{\phi}^{\omega} \phi\left(a_{k}\right)\right)\left(1_{A} *_{\phi}^{\omega} b_{k}\right)$

- $\zeta_{k}=Y_{k} Y_{k-1} \ldots Y_{1} w_{H} \xi$.

Then, as in the proof of Lemma 1.6, $\zeta_{1}$ is the vector $p_{L} a_{1} b_{1} \xi$ in the second component $L\left(=L^{+\otimes 0} \otimes L\right)$ of $K *^{\Omega} H$. 
By Lemma 1.6, for every $k, \zeta_{k}$ belongs to $\bigoplus_{j=0}^{k}\left(L^{+\otimes j} \otimes L\right)$, and thus is in the kernel of $w_{H}^{*}$. We have shown $w_{H}^{*} Y_{n} \ldots Y_{1} w_{H}=0$. An easy induction argument leads to the result that, for any $n \geqq 1, a_{1}, \ldots, a_{n}$ in $A, b_{1}, \ldots, b_{n}$ in $B$,

$$
w_{H}^{*}\left(a_{n} *_{\phi}^{\omega} 1_{B}\right)\left(1_{A} *_{\phi}^{\omega} b_{n}\right) \ldots\left(a_{1} *_{\phi}^{\omega} 1_{B}\right)\left(1_{A} *_{\phi}^{\omega} b_{1}\right) w_{H}
$$

lies in the range of $B$. As the $B$-module $H$ is faithful [we identify $B$ with its image in $\mathscr{L}(H)]$, we can write

$$
E_{\phi}^{\omega}(\alpha)=1_{A} *_{\phi}^{\omega}\left(w_{H}^{*} \alpha w_{H}\right), \quad \forall \alpha \in A *_{\phi}^{\omega} B .
$$

2) Let $q_{0}$ be the projection on $L^{\prime}=K \ominus \overline{A H}$, as a subspace of the second component $L$ of $K *^{\Omega} H$. For $n \geqq 1$, let $q^{n}$ be the projection on $L^{\prime+} \otimes L^{+\otimes(n-1)} \otimes L$, as a subspace $L^{+\otimes n} \otimes L$. $\left(L^{+}=K \Theta \overline{A H}^{-}:\right.$cf. 1.3). Then

- $q_{n}(n \geqq 0)$ commutes to the $a *_{\phi}^{\omega} 1_{B}(a \in A)$.

- For any $n \geqq 0$, any $k \geqq 0$, any $a_{1}, \ldots, a_{k} \in A$, any $b_{1}, \ldots, b_{k}, b_{k+1} \in B$ such that $\omega\left(b_{1}\right)=\ldots=\omega\left(b_{k}\right)=0$, then

$$
\begin{gathered}
q_{n}\left(1_{A} *_{\phi}^{\omega} b_{k+1}\right)\left(a_{k} *_{\phi}^{\omega} 1_{B}-1_{A} *_{\phi}^{\omega} \phi\left(a_{k}\right)\right) \ldots\left(a_{1} *_{\phi}^{\omega} 1_{B}-1_{A} *_{\phi}^{\omega} \phi\left(a_{1}\right)\right)\left(1_{A} *_{\phi}^{\omega} b_{1}\right) q_{n} \\
=(-1)^{k} \omega\left(b_{k+1}\right) \prod_{i=1}^{k} \omega\left(\phi\left(a_{k}\right) b_{k}\right) q_{n}
\end{gathered}
$$

(this comes from Lemma 1.6.2).

From these two properties, an easy induction argument shows that, for any $k \geqq 1$, any $a_{1}, \ldots, a_{k} A$, any $b_{1}, \ldots, b_{k}$ in $B$,

$$
q_{n}\left(a_{k} *_{\phi}^{\omega} 1_{B}\right)\left(1_{A} *_{\phi}^{\omega} b_{k}\right) \ldots\left(a_{1} *_{\phi}^{\omega} 1_{B}\right)\left(1_{A} *_{\phi}^{\omega} b_{1}\right) q_{n}
$$

lies in the range of $A$ in $\mathscr{L}\left(q_{n}\left(K *^{\Omega} H\right)\right)$.

As $q_{0}$ separates $A$, there exists a conditional expectation $E^{\prime}$ from $A *_{\phi}^{\omega} B$ into the range of $A$, such that

$$
\forall n \geqq 0, \quad \forall \alpha \in A *_{\phi}^{\omega} B, \quad q_{n} \alpha q_{n}=E^{\prime}(\alpha) q_{n} .
$$

Let us now prove the following property:

(1.7.3) Let $J$ be a bilateral ideal of $A *_{\phi}^{\omega} B$ which is both in the kernels of $E$ and $E^{\prime}$. Then $J=\{0\}$.

For $\alpha$ in $J$ satisfies $\alpha q_{n}=0, \forall n \geqq 0$ and $\alpha w_{H}=0$. Thus $\alpha a w_{H}=0, \forall a \in A$; thus $\alpha \eta=0$ for $\eta$ in $(\overline{A H} \cap L)=L \ominus L^{\prime}$; thus $\left.\alpha\right|_{L}=0$; thus $\left.\alpha b\right|_{L}=0, \forall b \in B$; thus $\left.\alpha\right|_{H^{-} \otimes L}=0$; thus $\left.\alpha a\right|_{H^{-} \otimes L}=0, \forall a \in A$; thus $\left.\alpha\right|_{\left(L^{+} \Theta L^{\prime+}\right) \otimes L}=0$; thus $\left.\alpha\right|_{L^{+} \otimes L}=0$; and so on.

We now see that (with the same $H$ ) when replacing $K$ by a smaller $K_{1}$ (but still $L_{1}^{\prime}=K_{1} \ominus \overline{A H}$ separating $A$ ), then we replace $A *_{\phi}^{\omega} B$ by a quotient algebra, but the kernel of the quotient map will be an ideal $J$ of elements $\alpha$ of $\left.A *_{\phi}^{\omega} B \operatorname{such} \alpha\right|_{H}=0$ and $\left.\alpha\right|_{L_{1}^{\prime}}=0$, thus $E_{\phi}^{\omega}(\alpha)=E^{\prime}(\alpha)=0$. By 1.7.3, the quotient map is faithful.

Same thing when replacing $H$ by a smaller $H_{1}$ (still containing $\Omega$ and separating $B$ ), without change of $L^{\prime}$. 


\section{Inductive Properties of $A *_{\phi}^{\omega} B$}

2.1. Let $A_{0}$ and $B_{0}$ be sub $C^{*}$-algebras of $A$ and $B$ respectively. Then $A_{0} *_{\phi}^{\omega} B_{0}$ will denote the sub $C^{*}$-algebra of $A *_{\phi}^{\omega} B$ generated by $\left\{a_{0} *_{\phi}^{\omega} 1_{B}, a_{0} \in A_{0}\right\}$ and $\left\{1_{A} *_{\phi}^{\omega} b_{0}\right.$, $\left.b_{0} \in B_{0}\right\}$. For instance, $A *_{\phi}^{\omega} \mathbb{C}_{B}$ and $\mathbb{C}_{A} *_{\phi}^{\omega} B$ are the canonical images of $A$ and $B$ respectively in $A *_{\phi}^{\omega} B$.

\subsection{Proposition}

$\left.1^{\circ}\right)$ Let $A_{1}$ and $B_{1}$ be $C^{*}$-algebras with unit; $\phi_{1}$ a completely positive map from $A_{1}$ into $B_{1}$, with $\phi_{1}\left(1_{A_{1}}\right)=1_{B_{1}} ; \varrho$ a representation (i.e. a $*$-homomorphism $\varrho\left(1_{A_{1}}\right)$ $=1_{A}$ ) of $A_{1}$ in $A, \pi$ a representation of $B_{1}$ in $B$ and $\omega_{1}$ a state on $B_{1}$ such that:

- $\omega \circ \pi=\omega_{1}$

- $\phi \circ \varrho=\pi \circ \phi_{1}$.

Then there exists a representation $\varrho * \pi$ from $A_{1} *_{\phi_{1}}^{\omega_{1}} B_{1}$ into $A *_{\phi}^{\omega} B$ characterized by:

- $(\varrho * \pi)\left(a_{1} *_{\phi_{1}}^{\omega_{1}} 1_{B_{1}}\right)=\varrho\left(a_{1}\right) *_{\phi}^{\omega} 1_{B}, \quad \forall a_{1} \in A_{1}$

$\cdot(\varrho * \pi)\left(1_{A_{1}} *_{\phi_{1}}^{\omega_{1}} b_{1}\right)=1_{A} *_{\phi}^{\omega} \pi\left(b_{1}\right), \quad \forall b_{1} \in B_{1}$

- $(\varrho * \pi) \circ E_{\phi_{1}}^{\omega_{1}}=E_{\phi}^{\omega} \circ(\varrho * \pi)$.

$\left.2^{\circ}\right)$ If both $\varrho$ and $\pi$ are injective, then $\varrho * \pi$ is also one to one.

Proof of the Proposition. Let $(\Omega, H, K)$ be a concrete realization of $(\phi, \omega)$ as defined in 1.2, with $H$ and $K$ satisfying conditions 1.7 (i) and (ii), so that $A *_{\phi}^{\omega} B$ is faithfully represented in $K *^{\Omega} H$.

Through $\pi$ and $\varrho, H$ is a left $B_{1}$-module and $K$ a $A_{1}$-module; $(\Omega, H, K)$ is also a concrete realization of $\left(\phi_{1}, \omega_{1}\right)$.

Let $\left(\Omega_{0}, H_{0}, K_{0}\right)$ be another concrete realization of $\left(\phi_{1}, \omega_{1}\right)$ satisfying 1.7 (i) and (ii) for $A_{1}$ and $B_{1}$. Define

$$
H_{1}=H \oplus H_{0}, \quad K_{1}=K \oplus K_{0},
$$

so that $A_{1} *_{\phi}^{\omega} B_{1}$ is faithfully represented in $K_{1} *^{\Omega} H_{1}$.

Let $V$ be the natural isometry from $K$ into $K_{1}$. It satisfies:

- $V \Omega=\Omega$

- $V p_{H}=p_{H_{1}} V$ (thus $\left.V(H) \subset H_{1}\right)$

- $V \varrho\left(a_{1}\right) \eta=a_{1} V \eta, \quad \forall \eta \in K, \quad \forall a_{1} \in A_{1}$

- $V \pi\left(b_{1}\right) \xi=b_{1} V \xi, \quad \forall \xi \in H, \quad \forall b_{1} \in B_{1}$,

and, with obvious notations

- $V p_{L}=p_{L_{1}} V$ (thus $\left.V(L) \subset L_{1}\right)$

- $V p_{L^{+}}=p_{L_{1}^{+}} V$ (thus $\left.V\left(L^{+}\right) \subset L^{+}\right)$.

Let $\tilde{V}$ be the isometry $\left.V\right|_{H} \otimes\left(V_{\mid L^{+}}\right)^{\otimes \mathbb{N}}$ from $K *^{\Omega} H=H \otimes L^{+\otimes \mathbb{N}}$ into $K_{1} *^{\Omega} H_{1}$ $=H_{1} \otimes\left(L_{1}^{+}\right)^{\otimes \mathbb{N}}$. Then $W \tilde{V} W^{*}$ is the isometry $V \otimes\left(V_{\mid L^{+}}\right)^{\otimes \mathbb{N}}$ from $K \otimes L^{+\otimes \mathbb{N}}$ into $K_{1} \otimes L_{1}^{+\otimes \mathbb{N}}$. 
We set $\varrho * \pi\left(\alpha_{1}\right)=\tilde{V}^{*} \alpha_{1} \tilde{V}, \forall \alpha_{1} \in A_{1} *_{\phi_{1}}^{\omega_{1}} B_{1}$, to obtain the conclusion of 1). For we have:

$$
\begin{array}{ll}
\tilde{V}^{*}\left(a_{1} *_{\phi_{1}}^{\omega_{1}} 1_{B_{1}}\right) \tilde{V}=\varrho\left(a_{1}\right) *_{\phi}^{\omega} 1_{B}, & \forall a_{1} \in A_{1} \\
\tilde{V}^{*}\left(1_{A_{1}} *_{\phi_{1}}^{\omega_{1}} b_{1}\right) \tilde{V}=1_{A} *_{\phi}^{\omega} \pi\left(b_{1}\right), & \forall b_{1} \in B_{1}
\end{array}
$$

and $\widetilde{V} w_{H} w_{H}^{*}=w_{H_{1}} w_{H_{1}}^{*} \tilde{V}$, so that, $\forall \alpha_{1} \in A_{1} *_{\phi_{1}}^{\omega_{1}} B_{1}$

$$
\begin{aligned}
\tilde{V}^{*}\left(E_{\phi_{1}}^{\omega_{1}}\left(\alpha_{1}\right)\right) \tilde{V} & =\tilde{V}^{*}\left(w_{H_{1}}^{*} \alpha_{1} w_{H_{1}} \otimes 1_{L^{+\otimes \mathbb{N}}}\right) \tilde{V} \\
& =\left[w_{H}^{*}\left(\tilde{V}^{*} \alpha_{1} \tilde{V}\right) w_{H}\right] \otimes 1_{L^{+\otimes \mathbb{N}}} \\
& =E_{\phi}^{\omega}\left(\varrho * \pi\left(\alpha_{1}\right)\right) .
\end{aligned}
$$

2) Is a consequence of 1.7.2, which implies that, when $\varrho$ and $\pi$ are faithful, $A_{1} *_{\phi_{1}}^{\omega_{1}} B_{1}$ is faithfully represented in $K *^{\Omega} H$.

2.3. Corollary. Let $A_{0}$ and $B_{0}$ be sub $C^{*}$-algebras of $A$ and $B$ respectively such that $\phi\left(A_{0}\right) \subset B_{0}$. Let $\phi_{0}$ and $\omega_{0}$ be the restrictions respectively of $\phi$ to $A_{0}$ and of $\omega$ to $B_{0}$.

$\mathrm{T}$ hen $\mathrm{E}_{\phi}^{\omega}\left(A_{0} *_{\phi}^{\omega} B_{0}\right) \subset \mathbb{C}_{A} *_{\phi}^{\omega} B_{0}$, and the $C^{*}$-algebras $A_{0} *_{\phi}^{\omega} B_{0}$ and $A_{0} *_{\varphi_{0}}^{\omega_{0}} B_{0}$ are canonically isomorphic.

2.4. Proposition. Let $A_{0}$ be a sub $C^{*}$-algebra of $A\left(1_{A} \in A_{0}\right)$ and $\varepsilon$ a conditional expectation from $A$ onto $A_{0}\left(\varepsilon\left(1_{A}\right)=1_{A}\right)$ such that $\phi \circ \varepsilon=\phi$. Then there exists a (unique) conditional expectation $\varepsilon^{*}$ from $A *_{\phi}^{\omega} B$ onto $A_{0} *_{\phi}^{\omega} B$ such that:

(i) $E_{\phi}^{\omega} \circ \varepsilon^{*}=E_{\phi}^{\omega}$

(ii) $\varepsilon^{*}\left(a *_{\phi}^{\omega} 1_{B}\right)=\varepsilon(a) *_{\phi}^{\omega} 1_{B}, \quad \forall a \in A$

(iii) $\forall n \geqq 1, \forall a_{1}, \ldots, a_{n} \in A, \forall b_{1}, \ldots, b_{n} \in B$, with $\omega\left(b_{2}\right)=\ldots=\omega\left(b_{n}\right)=0$,

$$
\varepsilon^{*}\left[\alpha_{n}\left(1_{A} *_{\phi}^{\omega} b_{n}\right) \ldots \alpha_{1}\left(1_{A} *_{\phi}^{\omega} b_{1}\right)\right]=0
$$

with

$$
\alpha_{n}=\left(a_{n}-\varepsilon\left(a_{n}\right)\right) *_{\phi}^{\omega} 1_{B}
$$

and

$$
\alpha_{k}=a_{k} *_{\phi}^{\omega} 1_{B}-1_{A} *_{\phi}^{\omega} \phi\left(a_{k}\right), \quad \forall k<n
$$

Proof of the Proposition. Set $\phi_{0}=\left.\phi\right|_{A_{0}}$, and let $\left(\Omega, H, K_{0}\right)$ be a concrete realization of $\left(\phi_{0}, \omega\right)$ satisfying 1.7 (i) and (ii).

Let $K$ be a $A$-module such that:

- $K_{0}$ is a closed subspace of $K$ and a sub $A_{0}$-module.

- $p_{K_{0}} a p_{K_{0}}=\varepsilon(a) p_{K_{0}}, \quad \forall a \in A$

- $L=K \Theta \overline{A H}$ is a faithful $A$-module.

Let $V_{0}$ be the identity isometry from $K_{0}$ into $K$, and $\tilde{V}_{0}=V_{0 I H} \otimes\left(V_{0 \mid L_{0}^{+}}\right)^{\otimes \mathbb{N}}$ be the induced isometry from $H *^{\Omega} K_{0}$ into $H *^{\Omega} K$ : as in the proof of Proposition 2.2, $\alpha_{0} \rightarrow \tilde{V}_{0}^{*} \alpha_{0} V_{0}$ is an isomorphism of $A_{0} *_{\phi}^{\omega} B$ onto $A_{0} *_{\phi_{0}}^{\omega} B$. 
What we have to show is the following property:

(2.4.1) $\forall n, b_{1}, \ldots, b_{n}, a_{1}, \ldots, a_{n}$ as in the setting of conclusion (iii) of the proposition, then

$$
\tilde{V}_{0}^{*} \alpha_{n}\left(1_{A} *_{\phi}^{\omega} b_{n}\right) \ldots \alpha_{1}\left(1_{A} *_{\phi}^{\omega} b_{1}\right) \tilde{V}_{0}=0
$$

For Property 2.4.1 will provide an implicit algorithm for calculating

$$
\tilde{V}_{0}^{*}\left(a_{n} *_{\phi}^{\omega} 1_{B}\right)\left(1_{A} *_{\phi}^{\omega} b_{n}\right) \ldots\left(a_{1} *_{\phi}^{\omega} 1_{B}\right)\left(1_{A} *_{\phi}^{\omega} b_{1}\right) \tilde{V}_{0}
$$

and verifying it lies in $A_{0} *_{\phi}^{\omega} B$. The existence of $\varepsilon^{*}=\tilde{\mathrm{V}}_{0}^{*} \cdot \tilde{\mathrm{V}}_{0}$ will be proved together with properties (i), (ii) and (iii); the unicity will be insured by the existence of such an algorithm.

Let us prove 2.4.1. We first write, for $k<n, \alpha_{k}=\alpha_{k}^{1}+\alpha_{k}^{2}$, with $\alpha_{k}^{1}=\left(a_{k}\right.$ $\left.-\varepsilon\left(a_{k}\right)\right) *_{\phi}^{\omega} 1_{B}$ and $\alpha_{k}^{2}=\varepsilon\left(a_{k}\right) *_{\phi}^{\omega} 1_{B}-1_{A} *_{\phi}^{\omega} \phi\left(a_{k}\right)$. As we have $\phi\left(a_{k}\right)=\phi\left(\varepsilon\left(a_{k}\right)\right)$, we have just to consider two cases: either $a_{k} \in \operatorname{ker} \varepsilon$, or $a_{k} \in A_{0}$.

For $\zeta_{0}$ in $K *^{\Omega} H$, set

$$
\zeta_{k}=\alpha_{k}\left(1_{A} *_{\phi}^{\omega} b_{k}\right) \ldots \alpha_{1}\left(1_{A} *_{\phi}^{\omega} b_{1}\right) \zeta_{0} .
$$

Consider these two situations:

a) $\zeta_{0} \in \operatorname{Im} \tilde{V}_{0}, a_{1}, \ldots, a_{k-1} \in A_{0}, a_{k} \in \operatorname{ker} \varepsilon$. Then $\left(1_{A} * b_{k}\right) \zeta_{k-1}$ lies in $\operatorname{Im} \tilde{V}_{0}$; $W\left(1_{A} * b_{k}\right) \zeta_{k-1}$ lies in $K_{0} \otimes L^{+\otimes \mathbb{N}}$ and $W \zeta_{k}$ lies in $\left(K \ominus K_{0}\right) \otimes L_{+}^{\otimes \mathbb{N}}$.

b) $\zeta_{0} \in\left(K \ominus K_{0}\right) \otimes L^{+\otimes \mathbb{N}} C L^{+} \otimes L^{+\otimes \mathbb{N}}, \quad a_{1}, \ldots, a_{k-1} \in A_{0}, \quad a_{k} \in \operatorname{ker} \varepsilon \quad$ and $\omega\left(b_{1}\right)=0$.

Then Lemma 1.6 insures that $\zeta_{k-1}$ lies in $L^{+} \otimes L^{+\otimes \mathbb{N}}$, so that $W\left(1_{A} *_{\phi}^{\omega} b_{k}\right) \zeta_{k-1}$ lies in $H^{-} \otimes L^{+\otimes \mathbb{N}} C K_{0} \otimes L^{+\otimes \mathbb{N}}$ : and again $W \zeta_{k}$ lies in $\left(K \ominus K_{0}\right) \otimes L^{+\otimes \mathbb{N}}$.

From a) and b), an easy induction argument leads to the result:

$$
W \zeta_{n} \in\left(K \ominus K_{0}\right) \otimes L^{+\otimes \mathbb{N}} \quad \text { if } \quad \zeta_{0} \in \operatorname{Im} \tilde{V}_{0}, \quad \text { and } \quad \tilde{V}_{0}^{*} \zeta_{n}=0 .
$$

As a consequence of the characteristic property 2.4 (iii) and the algorithm it provides for computing $\varepsilon^{*}$, we get the following corollary:

\subsection{Corollary}

1) In the situation of Proposition 2.4, let $A_{1}$ be a sub $C^{*}$-algebra of $A$ such that $\varepsilon\left(A_{1}\right) \subset A_{1}$. Then

(a) $\varepsilon^{*}\left(A_{1} *_{\phi}^{\omega} B\right) \subset A_{1} *_{\phi}^{\omega} B$.

(b) If $\varepsilon_{1}$ is the restriction of $\varepsilon$ and $\phi_{1}$ the restriction of $\phi$ to $A_{1}$, then, in the canonical identification of $A_{1} *_{\phi_{1}}^{\omega} B$ with $A_{1} *_{\phi}^{\omega} B$ (cf. Corollary 2.3), $\varepsilon_{1}^{*}$ identifies with the restriction of $\varepsilon^{*}$.

2) Let $\varepsilon_{1}$ and $\varepsilon_{2}$ be two conditional expectations in $A$ such that $\varepsilon_{1} \varepsilon_{2}=\varepsilon_{1}$, $\phi \circ \varepsilon_{1}=\phi$. Then $\varepsilon_{1}^{*} \varepsilon_{2}^{*}=\varepsilon_{1}^{*}$.

\section{Daniell-Kolmogorov Construction}

3.1. Theorem [Markov quantum semigroups admit quantum Markov dilations]. Let $A$ be a $C^{*}$-algebra with unit; let $\left(\phi_{t}\right)_{t \geqq 0}$ be a quantum Markov semigroup of $A$, that is a family $\left(\phi_{t}\right)_{t \in \mathbb{R}_{+}}$of completely positive maps of $A$ into itself indexed by the 
positive numbers (or any additive subsemigroup of $\mathbb{R}_{+}$) which satisfies:

$$
\begin{gathered}
\phi_{0}=\text { identity of } A \text { and } \phi_{s} \circ \phi_{t}=\phi_{s+t}, \quad \forall s, \quad t \in \mathbb{R}_{+}, \\
\phi_{s}\left(1_{A}\right)=1_{A}, \quad \forall s \in \mathbb{R}_{+} .
\end{gathered}
$$

Then there exists

- a $C^{*}$-algebra $\mathfrak{A}$ with unit, generated by a family $\left(\varrho_{t}\right)_{t \geqq 0}$ of faithful representations of $A$ into $\mathfrak{A}\left(\varrho_{s}\left(1_{A}\right)=1_{\mathfrak{A}}, \forall s \geqq 0\right)$

- for any $t \geqq 0$, a conditional expectation $E_{t}$ of $\mathfrak{A}$ onto $\mathfrak{A}_{t}$, the sub $C^{*}$-algebra of A generated by the $\left\{\varrho_{s}(A), s \leqq t\right\}\left[E_{t}\left(1_{\mathfrak{H}}\right)=1_{\mathfrak{H}}\right]$

- a time evolution $\left\{\sigma_{s}, s \geqq 0\right\}$ that is a semigroup of *-endomorphisms of $\mathfrak{A}$ $\left[\sigma_{s}\left(1_{\mathfrak{U}}\right)=1_{\mathfrak{U}} ; \sigma_{s} \circ \sigma_{t}=\sigma_{s+t}, \forall s, t \geqq 0 ; \sigma_{0}=\right.$ identity of $\left.\mathfrak{U}\right]$, with the following properties:

(i) $E_{s} E_{t}=E_{s}, \quad \forall s, \quad t \in \mathbb{R}_{+}, \quad s \leqq t$

(ii) $\sigma_{s} \circ \varrho_{t}=\varrho_{s+t}, \quad \forall s, \quad t \in \mathbb{R}_{+}$

(iii) $\sigma_{s} \circ E_{t}=E_{t+s} \circ \sigma_{s}, \quad \forall s, \quad t \in \mathbb{R}_{+}$(covariance rule)

(iii) $E_{s}\left[\sigma_{s}(\mathfrak{L})\right]=\varrho_{s}(A), \quad \forall s \geqq 0$ (weak Markov property)

(iv) $E_{0} \circ \varrho_{s}(a)=\varrho_{0}\left(\phi_{s}(a)\right), \quad \forall a \in A, \quad \forall s \in \mathbb{R}_{+}$.

Comments. Conclusion (i) means that the $\left\{\mathfrak{U}_{t}, E_{t}\right\}_{t \geqq 0}$ are an increasing filtration of $\mathfrak{A}$.

(ii) insures the coherence of the notations: $\varrho_{s}(A)$ can be interpreted as the algebra of events at time $s$ (the present at time $s$ ), and $\sigma_{s}$ as the time-evolution; the past of time $s$ will be $\mathfrak{A}_{s}=\bigvee_{t \leqq s} \varrho_{t}(A)$, and the future will be $\underset{t \geqq s}{\bigvee} \varrho_{t}(A)=\sigma_{s}(\mathfrak{I})$.

(iii) is covariance property. Written for $t=0$, it implies

$$
E_{s}\left[\sigma_{s}(\mathfrak{Q})\right]=\sigma_{s}\left[E_{0}(\mathfrak{R}) \mid=\sigma_{s}\left[\varrho_{0}(A)\right]=\varrho_{s}(A),\right.
$$

which is the weak Markov property (iii): all the information contained in the past $\mathfrak{A}_{s}$ and concerning the future $\sigma_{s}(\mathfrak{Q})$ in actually contained in the present $\varrho_{s}(A)$.

(iv) is dilation property; identifying $A$ with $\mathfrak{A}_{0}$ (through $\varrho_{0}$ ), it reads

$$
\phi_{s}=\left.E_{0} \circ \sigma_{s}\right|_{A}:
$$

the $*$-algebraic semigroup $\left\{\sigma_{s}\right\}_{s \geqq 0}$ dilates the quantum semigroup $\left\{\phi_{t}\right\}_{t \geqq 0}$.

Proof of the Theorem.

(3.1.1) Let $\Gamma=\left\{t_{1}, \ldots, t_{n}\right\} \quad\left(t_{1}<t_{2}<\ldots<t_{n}\right\}$ be a finite subset of $\mathbb{R}_{+}$. To $\Gamma$ is associated a $C^{*}$-algebra $\mathfrak{i}^{T}$, generated by $n$ (faithful) representations $\varrho_{t}^{T}(t \in \Gamma)$ of $A$, and a family $\left\{E_{t}, t \in \Gamma\right\}$ of conditional expectations of $\mathfrak{Q}^{\Gamma}$ onto the sub $C^{*}$-algebra $\mathfrak{U}_{t}^{T}$ generated by the $\left\{\varrho_{s}^{\Gamma}(A), s \leqq t\right\}$, through a decreasing induction process:

$$
\mathfrak{U}^{\Gamma}=\left(\ldots\left(\left(A *_{\phi_{t_{n}-t_{n-1}}}^{\omega} A\right) *_{\phi_{t_{n-1}-t_{n-2}}^{\omega}}^{\omega} A\right) *^{\omega} \ldots\right) * \stackrel{\omega}{\phi_{t_{2}-t_{1}}} A .
$$

- For $n=1$, we set $\mathfrak{U}^{\Gamma}=A, \varrho_{t_{1}}^{\Gamma}=$ identity

- For $n=2, \mathfrak{U}^{\Gamma}=A *_{\phi_{t_{2}-t_{1}}}^{\omega} A, \varrho_{t_{1}}^{\Gamma}=\left\{a \rightarrow 1_{A} *_{\phi}^{\omega} a\right\}$,

$$
\varrho_{t_{2}}^{\Gamma}=\left\{a \rightarrow a *_{\phi}^{\omega} 1_{A}\right\}, \quad E_{t_{1}}^{\Gamma}=E_{\omega}^{\phi}, \quad \text { with } \phi=\phi_{t_{2}-t_{1}} .
$$

- Let $\Gamma^{\prime}=\left\{\mathrm{t}_{2}, \ldots, \mathrm{t}_{n}\right\}$, and suppose you have constructed $\mathfrak{A}^{\Gamma^{\prime}}$, the $\varrho_{t}^{\Gamma^{\prime}}$, the $E_{t}^{\Gamma^{\prime}}$, $t \in \Gamma^{\prime}$. Then, identifying $\varrho_{t_{2}}^{\Gamma^{\prime}}(A)=E_{t_{2}}^{\Gamma^{\prime}}\left(\mathfrak{Q}^{\Gamma^{\prime}}\right)$ with $A$, we write

$$
\bar{\phi}=\overline{\phi_{t_{2}-t_{1}}}=\phi_{t_{2}-t_{1}} \circ E_{t_{2}}^{\Gamma^{\prime}}
$$


and set

$$
\begin{aligned}
& \cdot \mathfrak{A}^{\Gamma}=\mathfrak{A}^{\Gamma^{\prime}} *_{\phi_{t_{2}-t_{1}}}^{\omega} A \\
& \cdot \varrho_{t}^{\Gamma}=\left\{\begin{array}{lll}
\left\{a \rightarrow \varrho_{t}^{\Gamma^{\prime}}(a) * \frac{\omega}{\phi} 1_{A}\right\} & \text { if } t \in \Gamma^{\prime} \\
\left\{a \rightarrow 1_{\mathfrak{Q}} \Gamma^{\prime} * \frac{\omega}{\phi} a\right\} & \text { if } t=t_{1}
\end{array}\right. \\
& \cdot E_{t}^{\Gamma}=\left\{\begin{array}{lll}
\left(E_{t}^{\Gamma^{\prime}}\right)^{*} & \text { if } t \in \Gamma^{\prime} \\
E_{\bar{\phi}}^{\omega} & \text { if } & t=t_{1} .
\end{array}\right. \text { (cf. Proposition 2.2.1) }
\end{aligned}
$$

(3.2.1) Let $\Gamma$ and $\Gamma_{1}$ be finite subsets of $\mathbb{R}_{+}$, with $\Gamma_{1} \subset \Gamma$. Then there exists a faithful representation $\varrho_{\Gamma_{1}}^{\Gamma}$ of $\mathfrak{U}^{\Gamma_{1}}$ into $\mathfrak{U}^{\Gamma}$ which satisfies:

1) $\varrho_{\Gamma_{1}}^{\Gamma} \circ \varrho_{t}^{\Gamma_{1}}=\varrho_{t}^{\Gamma}, \quad \forall t \in \Gamma_{1}$,

2) $\varrho_{\Gamma_{1}}^{\Gamma} \circ E_{t}^{\Gamma_{1}}=E_{t}^{\Gamma} \circ \varrho_{\Gamma_{1}}^{\Gamma}, \quad \forall t \in \Gamma_{1}$.

This can be proved rather easily: by composition of the $\varrho_{\Gamma_{1}}^{\Gamma}$, we can suppose that $\Gamma$ has just one element more than $\Gamma_{1}$. If this element is the first one, we are in the situation of 3.1.1 above, and properties 1) and 2) come from the definitions.

If it is not the first one, we proceed from this element by a decreasing induction:

Let $\Gamma=\left\{t_{1}, \ldots, t_{n}\right\}, \Gamma^{\prime}=\left\{t_{2}, \ldots, t_{n}\right\}, \Gamma_{1}^{\prime}=\Gamma_{1} \cap \Gamma^{\prime}$; suppose you have got $\varrho_{\Gamma_{1}^{\prime}}^{\Gamma_{1}}$ faithful with properties $3.1 .2,1$ ) and 2 ); then you set

$$
\varrho_{\Gamma_{1}}^{\Gamma}=\varrho_{\Gamma_{1}^{\prime}}^{\Gamma_{1}^{1}} *_{\phi_{t_{2}-t_{1}}}^{\omega} i_{A} \quad \text { (Proposition 2.2.1), }
$$

which identifies $\mathfrak{U}^{\Gamma_{1}}$ with the sub $C^{*}$-algebra

$$
\mathfrak{U}^{\Gamma_{1}^{\prime}} *_{\phi}^{\omega} A \text { of } \mathfrak{U}^{\Gamma}
$$

property 1) is obvious, and property 2) is Corollary 2.5 (1). (3.1.3) We go now through the inductive limit by setting:

$$
\begin{gathered}
\mathfrak{U}=\lim _{\longrightarrow}\left\{\mathfrak{A}^{\Gamma}, \varrho_{\Gamma_{1}}^{\Gamma} ; \Gamma \in \mathscr{F}\right\} \\
\varrho_{t}=\lim _{\longrightarrow}\left\{\varrho_{t}^{\Gamma}, \Gamma \in \mathscr{F}\right\}, \quad E_{t}=\varliminf_{\lim }\left\{E_{t}^{\Gamma}, \Gamma \in \mathscr{F}\right\},
\end{gathered}
$$

where $\mathscr{F}$ is the filter of finite subsets of $\mathbb{R}_{+}$, ordered by inclusion.

Property (i) of the conclusion of Theorem 3.1 is an obvious consequence of 2.5 (2), and property (iv) results obviously from the construction and 1.7.1.

The existence of the time evolution $\sigma_{s}$ (which in this particular case will be one to one) comes from the construction in 3.1.1, which only depends on the differences $t_{k}-t_{k-1}$, and is thus invariant by time translation:

Let $\Gamma=\left\{t_{1}, \ldots, t_{n}\right\}$ and $\Gamma+s=\left\{t_{1}+s, \ldots, t_{n}+s\right\}$. Then there exists obviously an isomorphism $\sigma_{s}^{\Gamma}$ from $\mathfrak{A}^{\Gamma}$ into $\mathfrak{U}^{\Gamma+s}$ which satisfies:

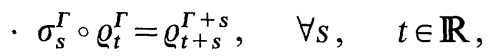

- $\sigma_{s}^{\Gamma} \circ E_{t}^{\Gamma}=E_{t+s}^{\Gamma+s} \circ \sigma_{s}^{\Gamma}$.

We get the $\left(\sigma_{s}\right)_{s \geqq 0}$ by going through the inductive limit. 


\section{References}

1. Accardi, L.: Non-commutative Markov chains associated to a preassigned evolution. Adv. Math. 29, 226-243 (1978)

2. Accardi, L.: Quantum stochastic processes. Preprint $n^{\circ} 17,1984$, Università di Roma II

3. Blumenthal, R.M., Getoor, R.K.: Markov processes and potential theory. New York: Academic Press 1968

4. Evans, D.E., Lewis, J.T.: Dilations of irreversible evolutions in algebraic quantum theory. Commun. Dublin Inst. Adv. Studies, ser. A, n 24, 1977

5. Hudson, R.L., Parthasarathy, K.R.: Construction of quantum diffusions. Quantum probability and applications to the quantum theory of irreversible processes. Lecture Notes in Mathematics Vol. 1055 Berlin, Heidelberg, New York: Springer 1982

6. Stinespring, W.F.: Positive functions on $C^{*}$-algebras. Proc. Am. Math. Soc. 6, 211-216 (1975)

7. Voiculescu, D.: Symmetries of some reduced free product $C^{*}$-algebras. Preprint, May 1983

8. Vincent-Smith, G.F.: Dilation of a dissipative quantum dynamical system to a quantum Markov process. Proc. Lond. Math. Soc. 49, 58-72 (1984)

Communicated by R. Haag

Received October 21, 1985; in revised form April 24, 1986 
